\title{
Dynamic Response of a Thermally Stressed Plate with Reinforced Edges
}

\author{
A.C.Santos Silva ${ }^{1} \cdot$ J. Lambros ${ }^{2}$ - D.M. Garner ${ }^{3}$ - E.A. Patterson ${ }^{1}$ (D) \\ Received: 10 April 2019 / Accepted: 8 July 2019 / Published online: 3 September 2019 \\ (C) The Author(s) 2019
}

\begin{abstract}
Conditions in hypersonic flight generate non-uniform temperature distributions that affect the performance and longevity of aircraft components. Historically, a limited amount of experimental work has been performed on the mechanical behaviour of reinforced aircraft structures subjected to very high temperatures. In this work on Hastelloy-X plates, non-contact techniques were used to measure the full-field temperature and deflection of a $1 \mathrm{~mm}$ thick plate with reinforced edges. The geometry was designed to emulate an aircraft's skin with the reinforced edges performing the function of stringers and ribs. High temperatures were achieved using quartz lamps arranged in various configurations with controllable power output. Digital image correlation (DIC) was used to measure surface displacements and a micro-bolometer mapped the temperature distribution across the plate. Deflection results for the reinforced plate showed it to behave as a dynamic system that buckles out-of-plane when heated before relaxing to a steady state. It is demonstrated that the out-of-plane displacement field experienced by the plate is influenced both by the in-plane temperature gradient and the energy supplied.
\end{abstract}

Keywords Reinforced plate $\cdot$ Dynamic response $\cdot$ High temperature $\cdot$ Plate deflection $\cdot$ Thermal buckling $\cdot$ Digital image correlation

\section{Introduction}

The behaviour of thin plates operating in high-temperature environments is relevant to a number of applications, including exhaust-wash structures associated with embedded engines in aircraft, reusable space vehicles, sustained hypersonic flight vehicles and breeder blankets in fusion nuclear reactors. Thin-gauge components, such as plates and panels used in these structures, are particularly affected by elevated temperatures that might induce a reduction or loss of structural stability as a result of the development of in-plane compressive stresses associated with the constraint of thermal expansion

\section{E. A. Patterson}

eann.patterson@liverpool.ac.uk

1 School of Engineering, University of Liverpool, The Quadrangle, Brownlow Hill, Liverpool L69 3GH, UK

2 Department of Aerospace Engineering, University of Illinois, 306 Talbot Laboratory, 104 S. Wright St., Urbana, IL 61801, USA

3 European Office of Aerospace Research and Development (EOARD), 86 Blenheim Crescent, Ruislip HA4 7HB, UK
[1]. In a limiting situation, these stresses reach a critical value leading to thermally-induced buckling.

Past analytical and computational studies focused on the influence of thermal stresses on the modal behaviour of plates. Mead [2] investigated, analytically and computationally, the effect of thermal stresses on the resonant modes and frequencies of free Kirchoff plates, which had been thermally stressed, and the results showed non-uniform temperature distributions to induce localised areas of compression and tension which influenced the dynamic behaviour of the structure. Chen and Virgin [3] confirmed these results for a simplysupported plate heated deeply into its post-buckled state, i.e. beyond the temperatures at which the plate buckles due to the heating. The authors showed vibrational mode switching to be associated with a change in the equilibrium configuration of the structure induced by the thermal buckling. More recent work, using computational mechanics, has included the development of models of functionally-graded plates resting on elastic foundations and subject to thermo-mechanical loading [4, 5]. Adineh and Kadkhodayan [5] applied a sudden uniform mechanical load to a simply-supported aluminium plate and their results showed the centre of the plate to spring-back after reaching a maximum out-of-plane displacement. This 
reflected earlier results for functionally-graded plates subjected to mechanical [6] and thermo-mechanical [7, 8] loading. As a combined result of thermal gradients through the thickness and the different elastic properties of the constituent materials of these structures, Vel and Batra [9] predicted a similar outof-plane dynamic response to a rapidly increasing thermal load. While, Steeves et al. [10] and, more recently Yamamoto et al. [11], have analysed the thermal behaviour of thin bimaterial structures tailored to provide specific coefficients of thermal expansion.

A limited amount of experimental analysis has been performed on thermally loaded plates using full-field measurement methods. The influence of thermally-induced compressive stresses on resonant frequencies and modal shapes were confirmed by Bailey [12] in a double-wedge square-cantilever and by Kaldas and Dickinson [13] who introduced a weld at the centre of a rectangular plate. Murphy et al. [14] used a centrally-located strain gauge and several thermocouples to monitor the behaviour of a rectangular stainless steel plate subjected to sinusoidal and broadband excitation while being heated. Other efforts have focused on fully-clamped or simply-supported plates and evaluated thermal buckling by plotting the central deflection as a function temperature [15-17]. Jin et al. [15] used digital image correlation to study a circular composite plate with its edges clamped using a titanium ring and subjected to a spatially uniform temperature distribution. Whilst Yuan et al. [16] took a similar approach when studying the critical buckling temperature and postbuckling behaviour of sandwich panels with truss cores. Thornton et al. [17] showed substantial out-of-plane bending to be strongly related to compressive membrane stresses caused by in-plane spatial temperature gradients. They used 15 linear variable differential transducers (LVDT) and 29 thermocouples to monitor displacements and temperatures across an unreinforced, simply-supported plate made from Hastelloy $\mathrm{X}$ and subjected to non-uniform heating. Pan et al. [18] presented analytical and experimental data that characterised the dynamic behaviour of the out-of-plane deflection in honeycomb sandwich panels used for thermal protection in hypersonic flight. Their thick, multi-material sandwich panels were placed in the vertical plane standing on an edge without any fixation and heated using infrared radiation on one side. Hence, from a review of the literature it can be concluded that there is a lack of experimental data describing the behaviour of geometrically-reinforced isotropic plates under thermal load and an attempt to bridge this gap is presented here. The aim of the current work is to establish a relationship between out-of-plane deflection and temperature non-uniformity during thermal loading of a thin plate with reinforced edges, and to do so by generating high-quality full-field temperature and displacement data that can be used both for understanding the plate response and validating detailed simulations.

\section{Experimental Procedure}

\section{Materials and Apparatus}

The study was conducted using a $250 \times 150 \mathrm{~mm}$ Hastelloy X (American Special Metals Inc., Miami, FL, USA) [19] plate with reinforced edges as shown in Fig. 1, vertically suspended using two stainless steel wires looped through two small holes in the plate's upper corners (also visible in the top corners of Fig. 1). The wires allowed the plate to hang from an aluminium portal frame bolted to an optical table and were sufficiently long and thin that there was negligible heat transfer through them. The specimen was manufactured by machining a $4.5 \mathrm{~mm}$ thick plate down to $1 \mathrm{~mm}$ thickness in the centre, while leaving a $10 \mathrm{~mm}$ wide frame of thickness $4.5 \mathrm{~mm}$ around its edges. The intention of the design was to represent the reinforcement in aircraft skins produced by stringers and ribs, which do not fully clamp the thin-gauge structure but constrain it by imparting a higher resistance to deformation. Several small holes were drilled in the reinforced edges so that multiple mounting options were available, but only one option was used in the results reported here.

The specimen was thermally loaded using arrays of halogen quartz lamps (QIR 2401000 V2D, Ushio, Steinhöring, Germany) with a maximum power output of $1 \mathrm{~kW}$ each and a colour temperature of $3210 \mathrm{~K}$. The lamps were uniformly spaced in an array of six as shown in Figs. 1 and 2 and their power output was regulated by a controller using TRIAC semiconductors. The specimen and lamps were not enclosed in a furnace so that there were substantial heat losses from the apparatus, but the optical access was excellent. The lamps were substantially longer than the plate's corresponding dimension (see Fig. 2) such that the energy flux from the lamps should be uniform over the transverse length of the plate, i.e. in the vertical direction.

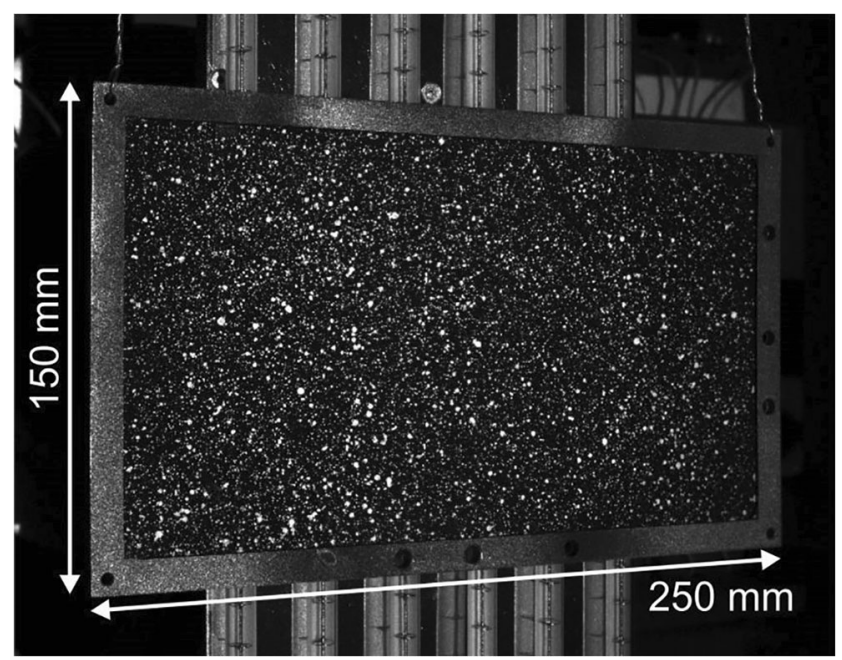

Fig. 1 Photograph of plate showing speckle pattern with quartz lamps behind the plate 


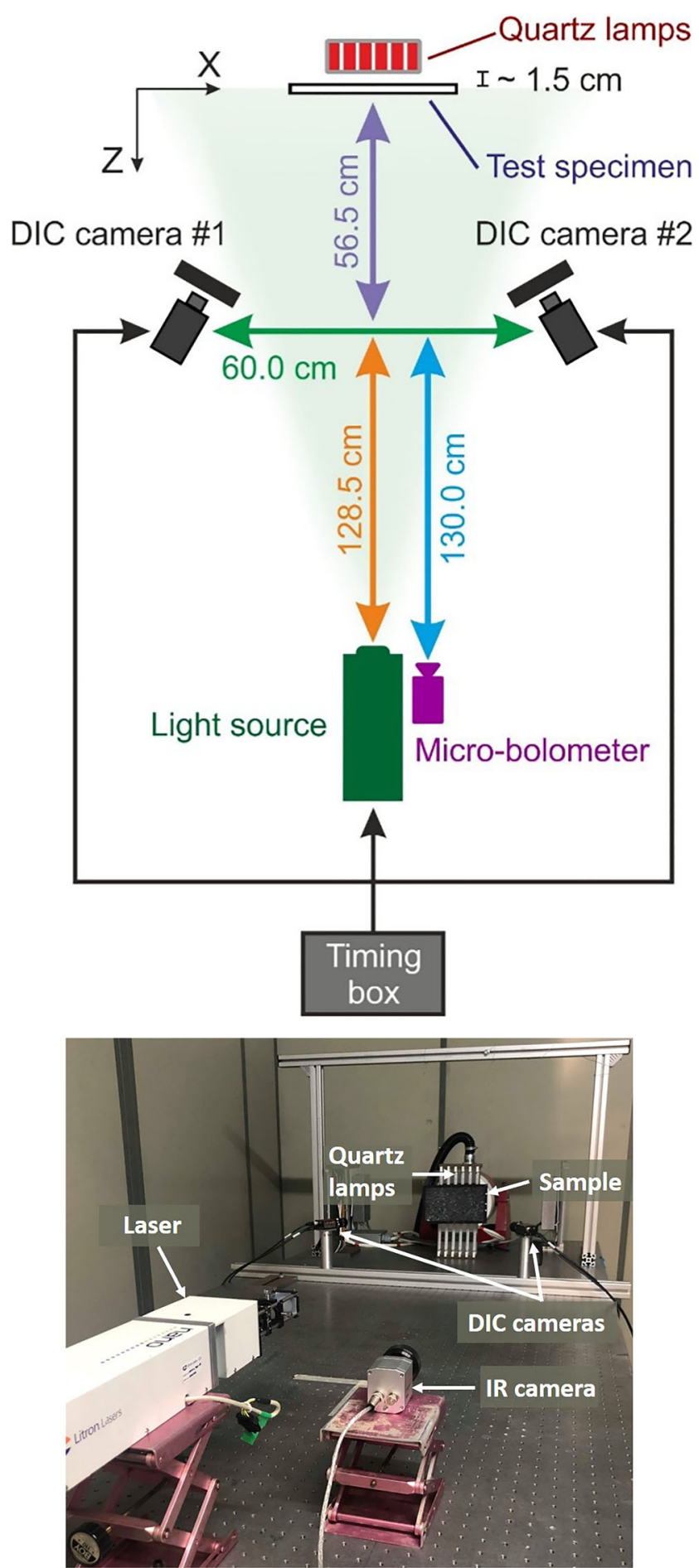

Fig. 2 Schematic (top) and photograph (bottom) of the test set-up

The full-field temperature distribution in the plate was monitored using an uncooled micro-bolometer (TIM 400, MICRO-EPSILON UK, Birkenhead, UK) with a $25 \times$ $25 \mu \mathrm{m}$ focal plane array of $382 \times 288$ pixels, which had been calibrated by the manufacturer in the $150-900{ }^{\circ} \mathrm{C}$ range and had an accuracy of $\pm 2{ }^{\circ} \mathrm{C}$ or $\pm 2 \%$ (whichever is greater). The instrument can be purchased with a calibration in the range $0-250{ }^{\circ} \mathrm{C}$ or $150-900{ }^{\circ} \mathrm{C}$; hence, a compromise was made to use a camera calibrated in the $150-900{ }^{\circ} \mathrm{C}$ range with an option selected that permitted its operation from $150{ }^{\circ} \mathrm{C}$ to room temperature. This resulted in a significant amount of noise for room temperature readings, which decreased the system's accuracy to $5-7^{\circ} \mathrm{C}$ (worst case scenario), according to the manufacturer. Since the work presented here is primarily focused on phenomena at temperatures beyond $150{ }^{\circ} \mathrm{C}$, the higher uncertainty of measurements at lower temperatures was accepted. A schematic and a photograph of the described experimental setup is shown in Fig. 2 which resulted in a spatial resolution of $1.1 \mathrm{~mm} /$ pixel for the micro-bolometer array.

The displacements of the thin section of the plate were measured using stereoscopic digital image correlation (DIC). The plate was prepared for high-temperature DIC by spraying a uniform layer of commercially-available black paint (VHT Flameproof, Cleveland, Ohio, USA) onto the front and back surfaces. A white variation of the same paint was used to create a random speckle pattern on the plate. The reverse colour scheme was considered but the use of black paint as the background was the preferred option because it allowed for larger absorption of radiation and, therefore, higher temperatures to be achieved in the specimen. The reinforced edges of the plate were painted black but not speckled, as the focus of this study was the thin section of the plate. At room temperature, the difference in emissivity of the white and black paint used in the DIC speckle yielded differences in apparent temperature that were below the resolution of the camera; so that, the speckle pattern was not visible in the infrared images from the micro-bolometer. As will be seen below, the scale of the temperature gradients when thermally loading the plate was much larger than the difference in readings imparted by the different paints.

The effect of temperature on the emissivity of the painted plate was analysed using a K-type thermocouple and comparing point-wise temperature measurements to those acquired with the micro-bolometer. Thermocouple measurements at different temperatures were plotted against local readings at the corresponding location from the micro-bolometer and a second-degree polynomial was fit to the data $\left(\mathrm{R}^{2}=0.9\right)$ and used to correct the full-field temperature maps.

A pulsed-laser digital image correlation (PL-DIC) system was used to acquire and process displacement fields during thermal loading. The system consisted of commercially-available stereoscopic DIC system (Q-400 system, Dantec Dynamics GmbH, Ulm, Germany) combined with a Nd:YAG laser (Nano L200-10, Litron, Rugby, England). The laser emitted a 4-ns pulse of light at $532 \mathrm{~nm}$ from which the speckle was removed using an 
optical Fourier filter and expanded to illuminate the entire specimen. High-quality images were acquired by two $1624 \times 1234$ pixel CCD Firewire cameras (2MP Stingray F-201b, Allied Vision Technologies GmbH, Stradtroda, Germany) fitted with optical bandpass filters with a centre wavelength of $532 \mathrm{~nm}$ and bandwidth of $4 \mathrm{~nm}$, i.e. corresponding to the laser illumination, so that cameras were not blinded by the lamps providing the heating. The maximum frame rate of the cameras was 14 frames per second at the full resolution of $1624 \times 1234$ pixels, which corresponded to an average spatial resolution of $0.23 \mathrm{~mm} /$ pixel. Synchronisation of the PL-DIC system was achieved by using a timing box that provided a trigger signal for both the laser and the CCD cameras. Image processing and calculation of data fields of out-of-plane displacement was performed using the Istra 4D software supplied with the system, employing square facets of 25 pixels $(5.75 \mathrm{~mm})$ with a 4 pixel $(0.92 \mathrm{~mm})$ overlap that corresponded to the pitch of the displacement data.

Prior to any experiments, the plate was thermally loaded for 10 min using six lamps at full power, symmetrically positioned about the plate's centre. This procedure was followed to relieve some of the residual stresses introduced by the manufacturing of the specimen and created an initial curvature of the plate that was convex towards the lamps, as shown in Fig. 3, which better represents real components that are rarely flat. As seen by Thornton et al. [17], this initial curvature determined the direction of deformation in the following experiments.

Two types of experiments were conducted, in which the energy supplied to the plate was incremented: in the first set of experiments, different numbers of lamps were used in the thermal loading of the specimen; whilst the second set involved heating the plate with a constant number of lamps whilst controlling their power output. These heating regimes

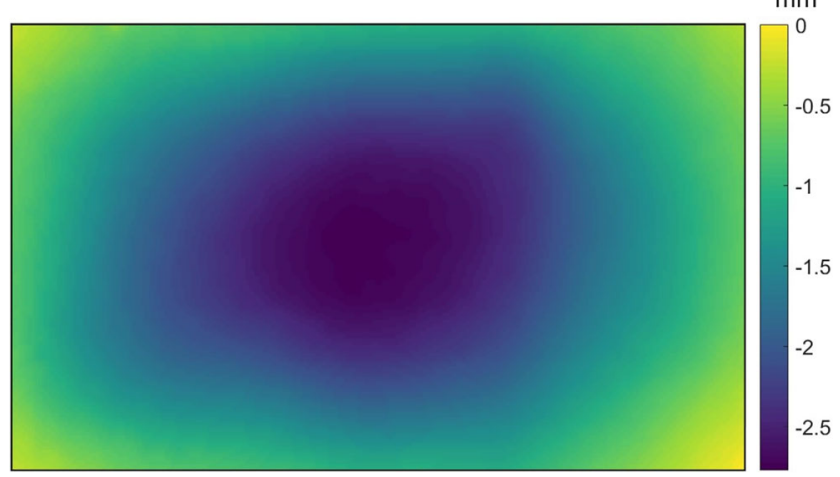

Fig. 3 Initial shape of the thin plate at room temperature calculated from DIC measurements using Istra 4D following an initial thermal cycle corresponding to heating with six lamps at full power for $10 \mathrm{~min}$ from room temperature and then allowing the plate to cool in air to room temperature. The shape is shown as z-direction displacements from the $\mathrm{x}-\mathrm{y}$ plane
Fig. 4 Steady state temperature measurements for the thin section of the reinforced plate subjected to non-uniform heating using a variable number of lamps at full power (left) and nominally uniform heating using six lamps (right); with constant rates of energy supplied on each row but decreasing from top to bottom

resulted in non-uniform temperature distributions and nearuniform temperature distributions, respectively, which are shown in Fig. 4. Experiments were limited to 10 min duration to avoid accumulating changes due to material aging, which occurs when heating to temperatures around $760{ }^{\circ} \mathrm{C}$ for approximately $3 \mathrm{~h}$ [20]. Similarly, all experimental temperatures were kept below the recrystallisation or critical temperature of the material $\left(1163-1191{ }^{\circ} \mathrm{C}\right)$ to avoid annealing or solution treating [20]. After each experiment, the specimen was allowed to air cool to room temperature.

To estimate the noise floor for the out-of-plane measurements, a series of 30 images were acquired with the panel in a static configuration at room temperature and then processed using Istra 4D. The mean value of the out-of-plane displacement component was then calculated and the noise floor was found to be approximately $16.6 \mu \mathrm{m}$. There were some concerns that local variations in the refractive index of the air between the specimen and cameras would introduce noise into the digital image correlation; however, Pan et al. [21] observed that this did not occur when the specimen is vertical and perpendicular to the optical axis, as was the case in this study. Hence, no discernible local variation in refractive index were observed.

\section{Experiments on Non-uniform Temperature Distributions}

The suspended reinforced plate was thermally stressed using successively six, four, two and one quartz lamps, with their axes parallel to the short side of the plate. The lamps were uniformly distributed with a centre-to-centre spacing of $3.5 \mathrm{~cm}$ and positioned symmetrically about the plate's transverse and longitudinal axes. DIC and temperature data acquisition began at room temperature after which the lamps were switched on at full power and after about $200 \mathrm{~s}$ the plate reached a stable temperature distribution. The acquisition frequencies were $1 \mathrm{~Hz}$ and $4 \mathrm{~Hz}$ for the temperature data and DIC images respectively. In order to estimate the error associated with the measurements, the same acquisition method for fullfield temperature and DIC data was used when transversely loading the plate six times using 2 lamps at full power. The standard deviations of the datasets for the displacements and temperatures at the mid-point (facet for the displacements) of the plate were calculated and are shown in Fig. 5. In addition, for each repeat, the location of the facet where the maximum displacement occurred was identified and this location varied 
6 lamps $\equiv$ Qin

\section{6 lamps @ Qin}

${ }^{\circ} \mathrm{C}$
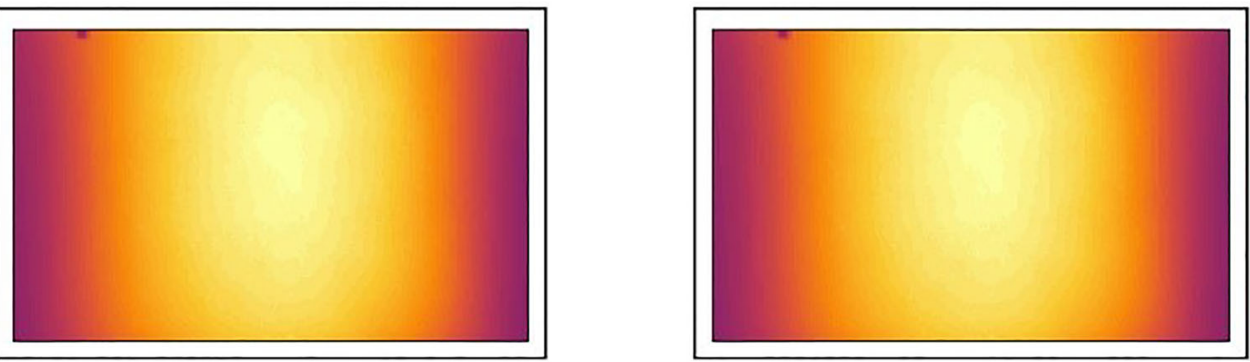

695

600

4 lamps $\equiv 0.57$ Qin 6 lamps @ 0.57 Qin
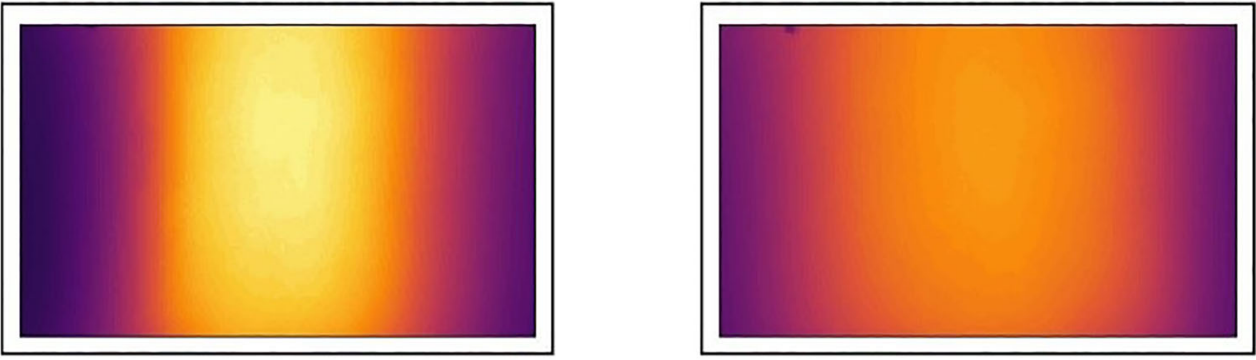

500

400

2 lamps $\equiv 0.27 \dot{Q}$ in 6 lamps @ 0.27 Qin
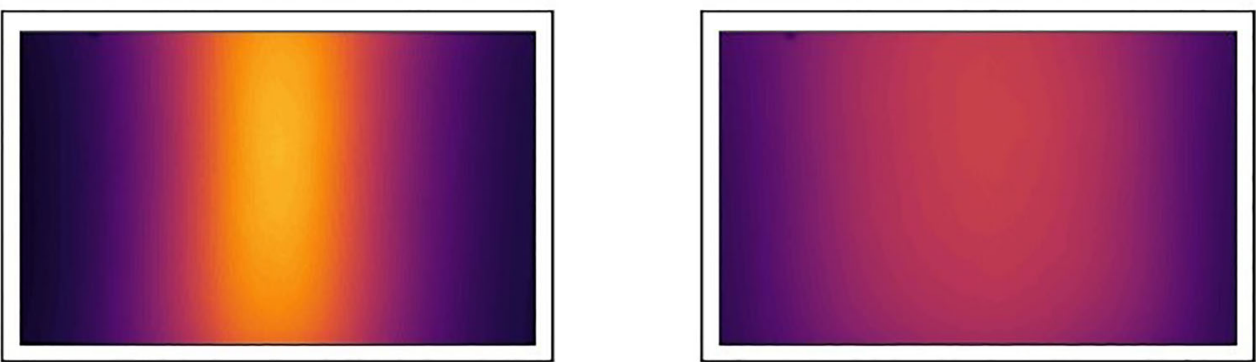

300

\section{1 lamp $\equiv 0.06 \dot{Q}$ in 6 lamps @ 0.06 Qin}

200
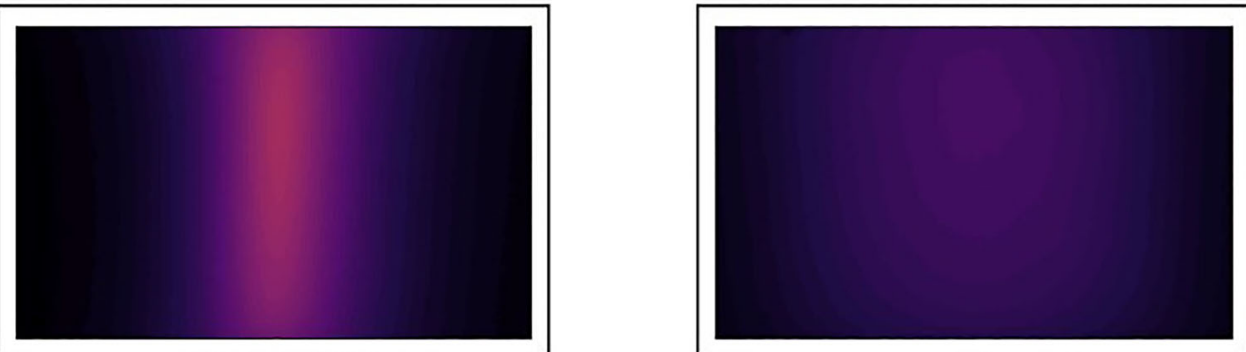

100

33 


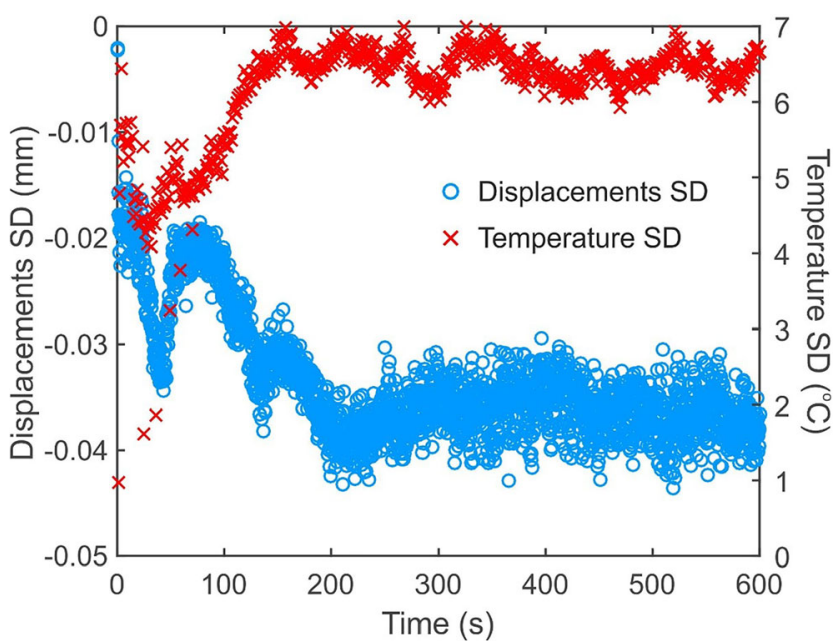

Fig. 5 Standard deviation (SD) of the displacement (left axis and blue markers) and temperature (right axis and red markers) measurements at the mid-point of the thin plate based on six repetitions of heating the plate to a steady state using two lamps at full power

by no more than a vector pitch $(4.83 \mathrm{~mm})$ in the transverse and longitudinal directions.

In order to facilitate comparison with results from previous investigations [1-3], the Istra software was used to remove rigid body motions prior to selecting the data for the out-ofplane deflection at the centre of the plate and plotting it as a function of time together with the corresponding point-wise temperature results at the centre of the plate for each thermal loading scenario in Fig. 6(a).

\section{Experiments on Uniform Temperature Distributions}

The use of a different numbers of lamps, in the previous section, produced a series of non-uniform spatial distributions of temperature along the longitudinal axis of the plate; and, the energy supplied to the plate $\left(Q_{i n}\right)$ was also changed by the number of lamps used. Hence, to better understand the observed behaviour, experiments were conducted using the maximum number of lamps to achieve an approximately uniform temperature distribution; but, adjusting the energy supply to the lamps so that $Q_{i n}$ corresponded to values in the previous set of experiments. Six lamps were used in this set of experiments, and the energy output of each lamp was defined as a function of the rate of energy supplied to the plate at steady state, $\dot{Q}_{i n}$ when using six, four, two and one lamps. It was assumed, for steady state conditions, that the heat transfer from the plate to the environment was equal to heat supplied, $\dot{Q}_{i n}$. This heat transfer was evaluated using the average temperature of the plate calculated using a surface integral fitted to the steady-state, full-field temperature measurements using the nearest neighbour method in MATLAB. The conduction heat losses through the hanging wire were found to be negligible and so, the sum of the steady-state convection and radiation heat transfer rates were equated to the unknown rate of heat supplied, $\dot{Q}_{i n}$. The reinforced edges were excluded from this analysis from which a calibration graph for the rate of heat supplied, $\dot{Q}_{i n}$, against controller settings was obtained using the temperature data collected with six lamps; and this graph was used to identify controller settings for the six lamps that yielded an approximate rate of heat supplied, $\dot{Q}_{i n}$ comparable to four, two and one lamps at full power. These settings were deployed using the same experimental methodology and data analysis as described in 2.1. The resultant out-of-plane deflection and temperature data are plotted in Fig. 6(b).

\section{Results and Discussion}

The temperature fields and corresponding fields of z-direction displacement, relative to the initial shape of the plate, measured in each experiment in the steady-state after $10 \mathrm{~min}$ are presented in Figs. 4 and 7, respectively. In all cases, the plate is suspended from wires at its top corners and hence the displacement at these corners is nominally zero; however, the $\mathrm{z}$-displacement in the bottom corners has positive values varying from close-to-zero to approximately $1.5 \mathrm{~mm}$ as the plate rotates about its attachment points to counteract the buckling of its centre in the negative z-direction, i.e. to keep its centroid in the same $x-y$ plane as the attachments. For a constant number of lamps supplying energy, the out-of-plane displacement field increases in magnitude with the rate of energy supplied, $\dot{Q}_{\text {in }}$ to the plate but without a significant change in shape (right column in Fig. 7). However, the response was different when the number of lamps was varied (left column in Fig. 7) because both the magnitude and shape of the displacement field changed with heat supplied.

The measured displacement fields are unlikely to contain significant errors because it has been previously shown that the errors associated with the PL-DIC system are less than $4 \%$ for dynamic events [22]. Room temperature measurements of the curvature of the plate were made before and after each thermal loading and the difference found to be below the noise floor of the setup $(16.6 \mu \mathrm{m})$. Therefore, it was concluded that the shape of the plate had not significantly changed as a consequence of each loading scenario. The plate appeared to follow the same deformation path under heating and cooling conditions.

The point-wise analysis of the out-of-plane displacement at the centre facet of the plate is presented in the top row of Fig. 6 as a function of time and shows a dynamic response to the thermal loading. When the temperature reaches a constant value, the rate of plate deformation reverses and the plate relaxes to a steady-state position with a spring-back of the order $40 \%$ for heating with six lamps at full power. This behaviour is present for all lamp and power configurations in 
a
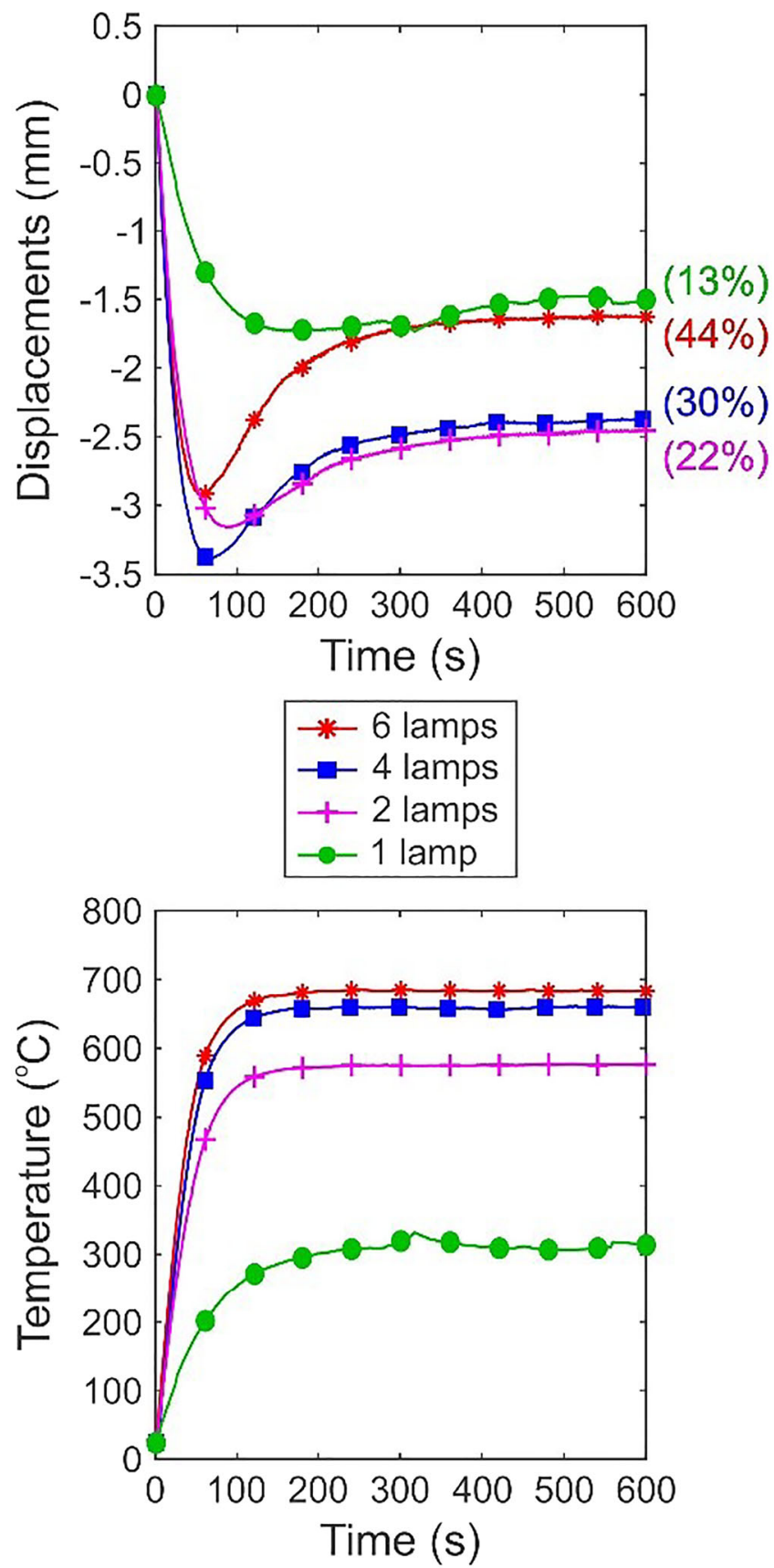

b
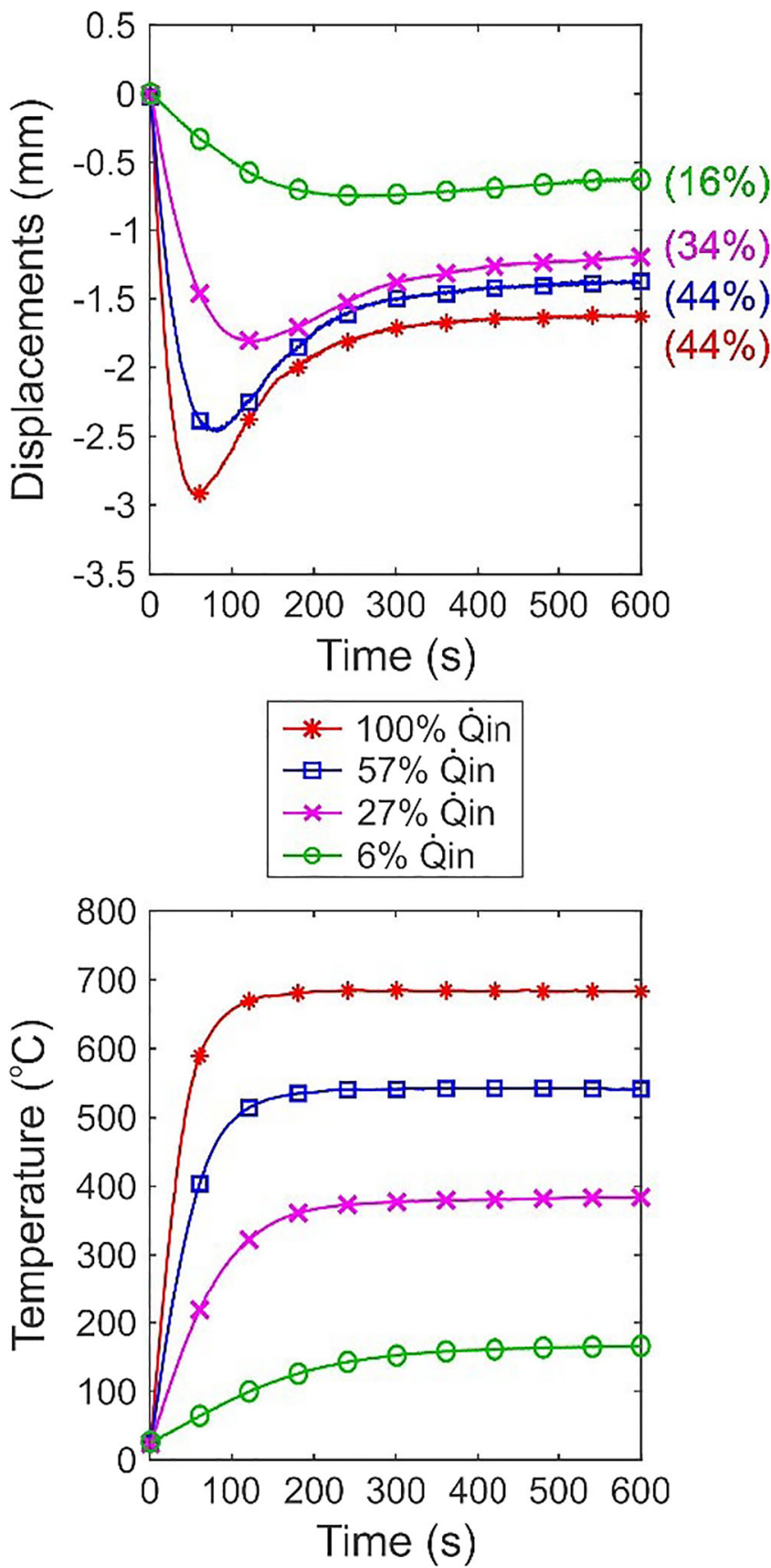

Fig. 6 Point-wise displacement at the centre of the plate (top) and corresponding temperature measurements (bottom) as (a) a function of the number of lamps (non-uniform heating) and (b) the power output of six lamps (nominally uniform heating). For illustrative purposes, symbols were only included with the plotted lines at $60 \mathrm{~s}$ intervals. The level of central spring-back is shown in brackets in the displacement plots and the displacements are relative to the initial shape of the plate shown in Fig. 3 (convex towards the lamps) and exclude rigid body motion

Fig. 4, except for the lowest level of heat supplied to the plate, i.e. one lamp at full power and six lamps at $6 \%$ of $\left(\dot{Q}_{i n}\right)$. In these two low energy cases, the rate of temperature increase with time was substantially lower than in the other cases and asymptotically approached a constant value with the result that the plate relaxes gently to a deformation of $13 \%$ to $16 \%$ lower than the maximum deflection. For the experiment conducted with a single lamp at full power, there appeared to be have been some fluctuation of lamp power output, which is evident in the temperature and DIC measurements as small perturbations. The estimates of uncertainty in Fig. 5 show the standard deviation of both displacement and temperature 


\section{6 lamps $\equiv$ Qin}

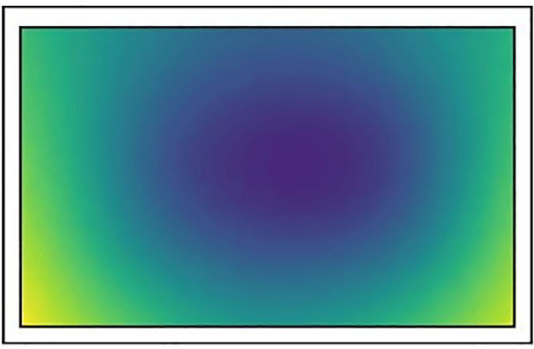

\section{4 lamps $\equiv 0.57 \dot{Q}$ in}

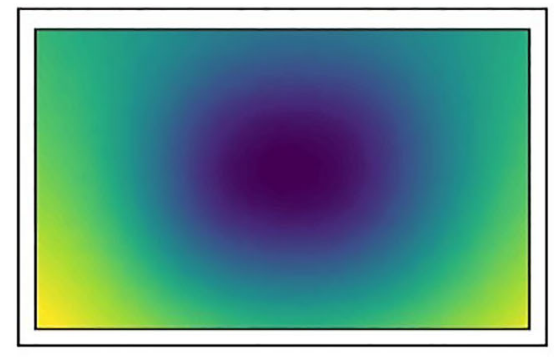

\section{6 lamps @ Qin}

\section{2 lamps $\equiv 0.27$ Qin}

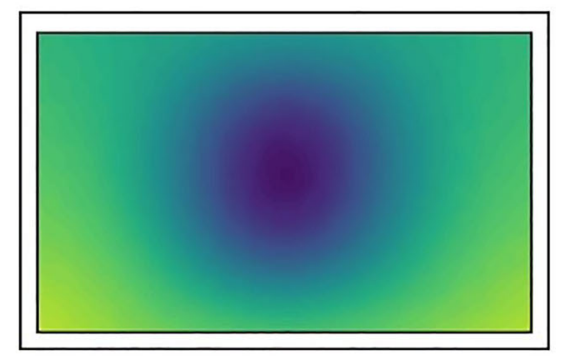

\section{1 lamp $\equiv 0.06 \dot{Q}$ in}

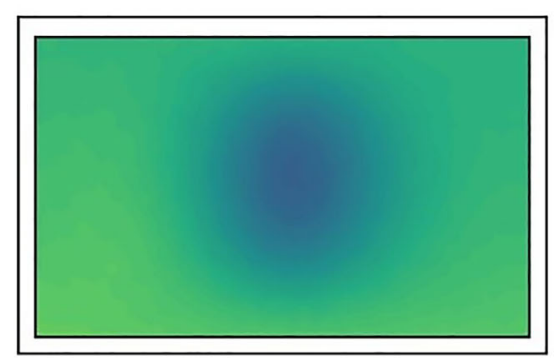

Fig. 7 Steady-state measured out-of-plane displacements (including rigid body motion and relative to the initial shape in Fig. 4) for the thin section of the reinforced plate subject to the thermal loads characterised by the temperature measurements shown in Fig. 4

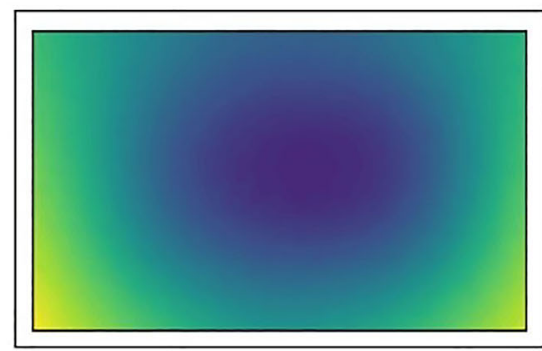

6 lamps @ 0.57Q்in

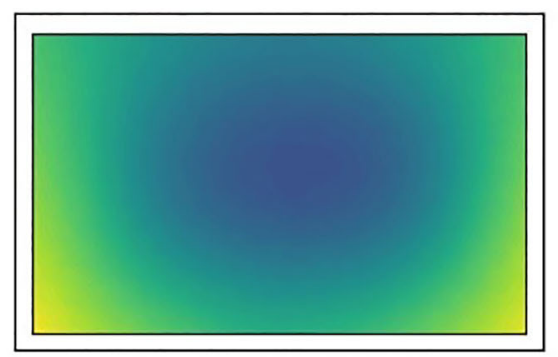

\section{$\mathrm{mm}$}

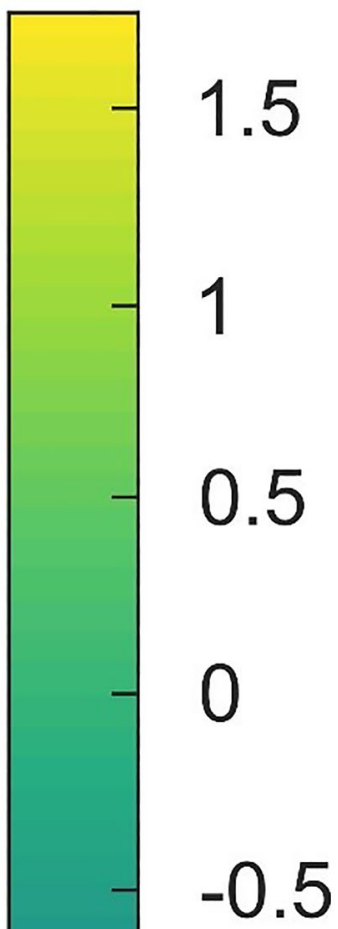

\section{6 lamps @ 0.27Q்in}

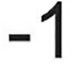

$-1.5$

$-2$

$-2.5$

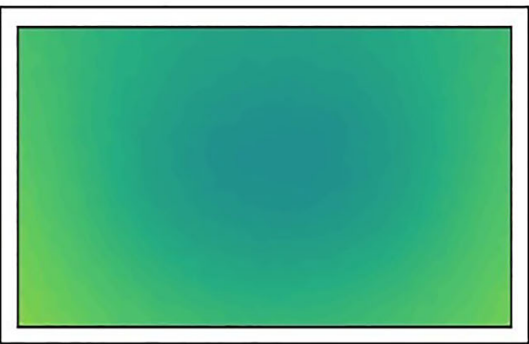


a

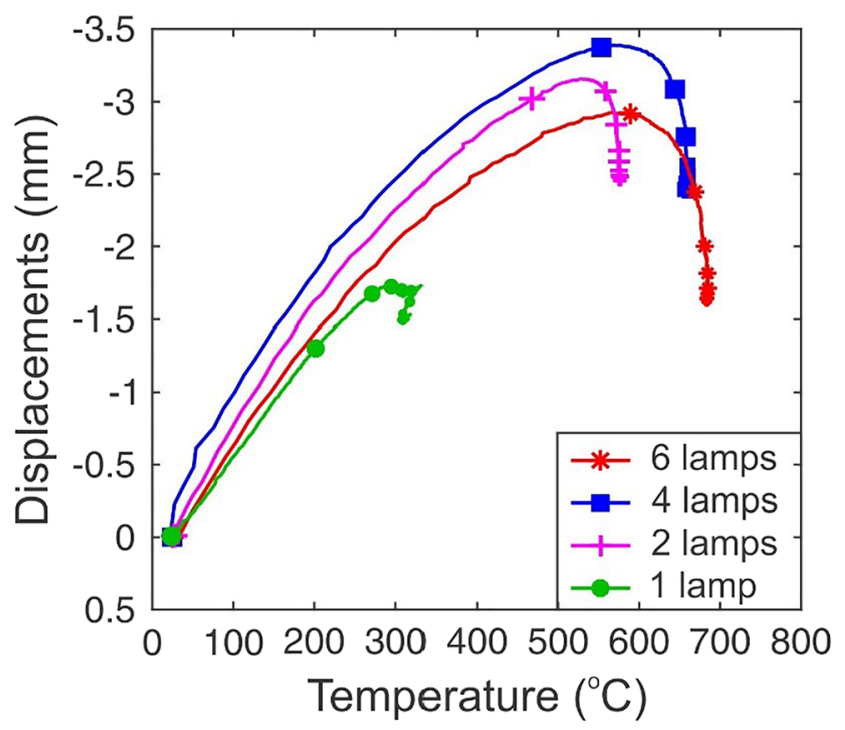

b

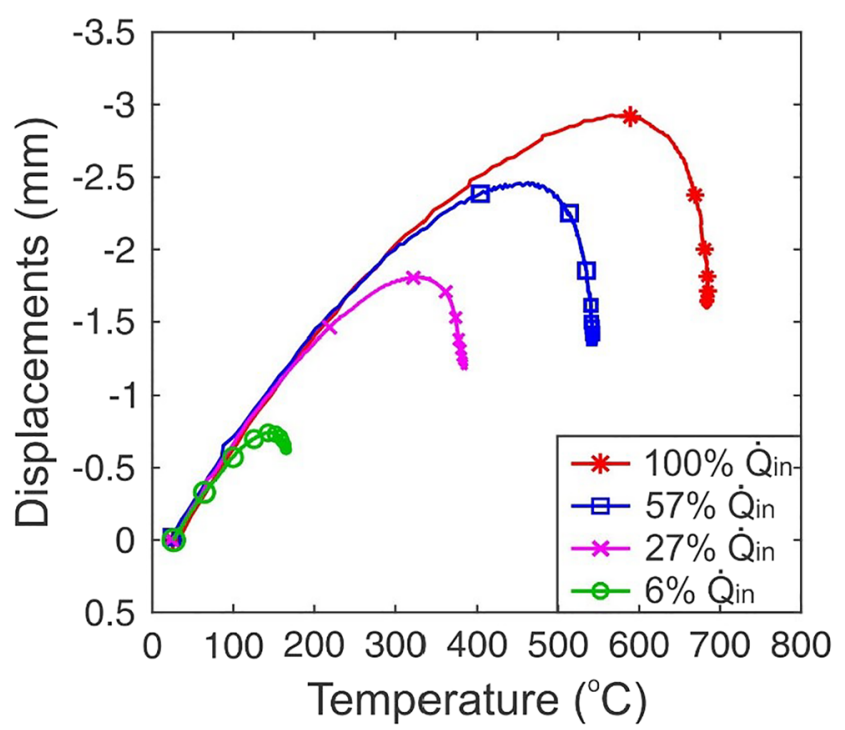

Fig. 8 Point-wise displacement values at the centre of the plate as a function of the corresponding temperature when heating the specimen as a function of (a) the number of lamps and (b) the power output of six lamps. For illustrative purposes, symbols were only included with the plotted lines at $60 \mathrm{~s}$ intervals of loading. The displacements are relative to the initial shape of the plate shown in Fig. 3 (convex towards the lamps) and exclude rigid body motion

readings at the centre of the plate to be below $45 \mu \mathrm{m}$ and $7{ }^{\circ} \mathrm{C}$, respectively, which supports the conclusion that the differences between the experiments, in terms of the displacement and temperature measurements in Fig. 6, are significant.

The temperature data in Fig. 6 (bottom) were plotted as a function of the displacement measurements in Fig. 6 (top) and the results are shown in Fig. 8. For both sets of experiments, the gradients of these graphs for the first $10 \mathrm{~s}$ are within 1.5 standard deviations of the mean of each set of experiments,
Table 1 Measured rate of temperature change and displacement at the centre of the plate for each condition in the first $10 \mathrm{~s}$ of heating with rigid body motion excluded from the displacement measurements

\begin{tabular}{lllll}
\hline $\begin{array}{l}\text { Nominal } \\
\text { temperature } \\
\text { distribution }\end{array}$ & $\begin{array}{l}\text { Heating } \\
\text { condition }\end{array}$ & $\begin{array}{l}\text { Initial rate } \\
\text { of } \\
\text { temperature } \\
\text { change } \\
\left({ }^{\circ} \mathrm{C} / \mathrm{s}\right)\end{array}$ & $\begin{array}{l}\text { Initial } \\
\text { displacement } \\
\text { rate }(\mu \mathrm{m} / \mathrm{s})\end{array}$ & $\begin{array}{l}\text { Initial } \\
\text { displacement/ } \\
\text { temperature } \\
\text { gradient } \\
\left(\mu \mathrm{m} /{ }^{\circ} \mathrm{C}\right)\end{array}$ \\
\hline Non-uniform & 6 lamps & $13.6 \pm 5.1$ & $117.6 \pm 12.7$ & $8.6 \pm 2.5$ \\
& 4 lamps & $10.7 \pm 5.1$ & $127.7 \pm 12.7$ & $11.5 \pm 2.5$ \\
& 2 lamps & $9.7 \pm 5.1$ & $97.9 \pm 12.7$ & $10.1 \pm 2.5$ \\
& 1 lamp & $4.0 \pm 5.1$ & $25.6 \pm 12.7$ & $6.4 \pm 2.5$ \\
& Mean & $9.5 \pm 5.1$ & $92.2 \pm 12.7$ & $9.2 \pm 2.5$ \\
& 100\% & $13.6 \pm 5.1$ & $117.6 \pm 12.7$ & $8.6 \pm 2.5$ \\
& Qin & & & \\
& 57\% Qin & $7.7 \pm 5.1$ & $72.0 \pm 12.7$ & $9.4 \pm 2.5$ \\
& 27\% Qin & $3.5 \pm 5.1$ & $32.2 \pm 12.7$ & $9.1 \pm 2.5$ \\
& 6\% Qin & $0.4 \pm 5.1$ & $5.3 \pm 12.7$ & $10.8 \pm 2.5$ \\
& Mean & $6.3 \pm 5.1$ & $56.8 \pm 12.7$ & $9.5 \pm 2.5$ \\
\hline
\end{tabular}

see Table 1; i.e., varying the power or the number of lamps does not have a significant effect on the initial rate of change in the central displacement with temperature. Figures 6(a) and 8(a) show that the largest central displacement occurred when using four lamps at full power, with the next largest being for two lamps at full power. Hence, the central deflection of the reinforced plate under thermal load was not proportional to the rate of energy supplied when the spatial distribution of the energy supplied was also changed. A comparison of the results in Fig. 8(a) and (b) suggests that the larger central deflections are associated with thermal loading of a narrower region of the plate and, therefore, a steeper gradient in the spatial distribution of temperature in the longitudinal direction. This agrees with early analytical work by Mansfield [23] and computational predictions by Liu et al. [4] that suggested the buckling temperature of a composite plate decreased with an increasing in-plane temperature gradient. A likely explanation of this behaviour is that the maximum temperature in the four-

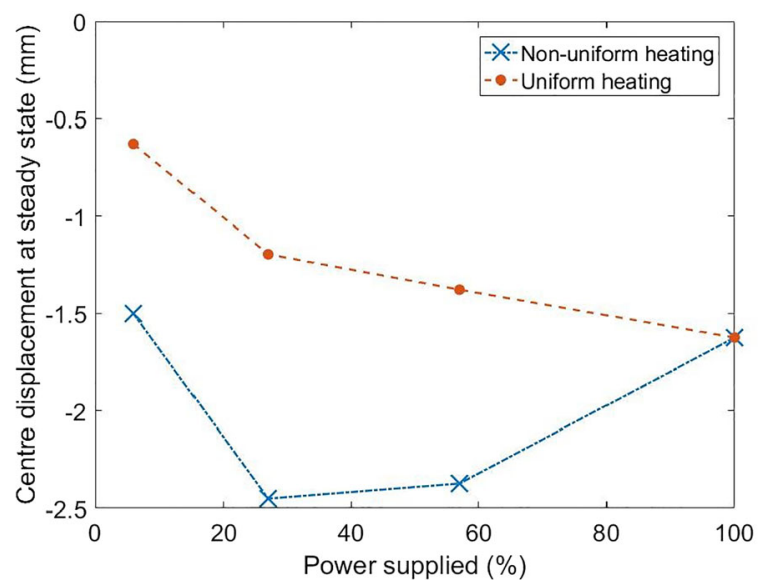

Fig. 9 Point-wise displacement of the centre of the plate as a function of power supplied. The measurements presented were acquired at steady state and rigid body motion has been removed 
Fig. 10 Point-wise temperature measurements at the centre and edges of the plate (top) when heating the structure with 2 lamps which results in the temperature distributions shown (bottom) that include the reinforced edges. The temperature differences between the point locations shown in the top diagram are also shown. The central displacement of the plate with rigid body motion removed is provided for comparative purposes and symbols are shown for illustrative purposes only

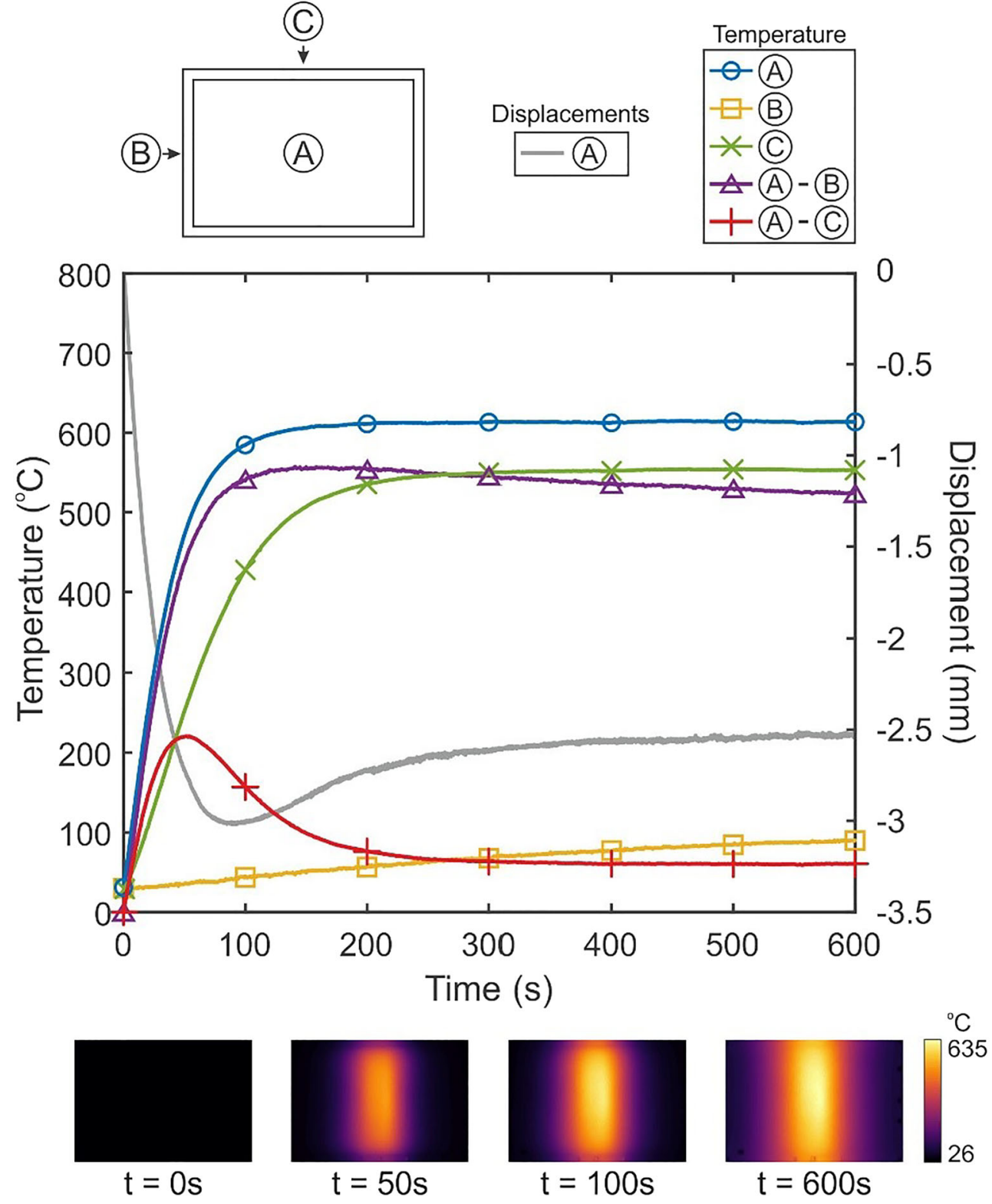

lamp case is approximately the same as the six-lamp case (see Fig. 6 bottom left panel); so, the local reduction in modulus and local thermal expansion will be approximately the same in the centre. However, the temperature towards the edge of the plate is much lower in the four-lamp case (see Fig. 4 top left two panels) so that the local reduction in modulus will be smaller leading to a higher level of constraint and this renders a local out-of-plane deflection at the centre more likely in the four-lamp case, as seen in the measurements (see Fig. 8). Note that the dynamic modulus of elasticity of Hastelloy $\mathrm{X}$ reduces from $205 \mathrm{GPa}$ at room temperature to $161 \mathrm{GPa}$ at $760{ }^{\circ} \mathrm{C}$ [19], which is a $20 \%$ reduction; while the mean coefficient of thermal expansion increases by about $13 \%$, from about 13.9 to $15.8 \mathrm{~m} / \mathrm{m}{ }^{\circ} \mathrm{C}[19]$, over the same range.
However, in experiments using nominally uniform heating, the location and number of the heat sources was not changed; hence, the same area of the plate was heated in every case, causing the shape of the temperature fields shown in Fig. 4 to remain similar. In this scenario, the central displacement is proportional to the power supplied, except at the lowest level of power supplied, as shown in Fig. 9. These results for the out-of-plane deflection at the centre of an isotropic plate with reinforced edges are similar those observed by Pan et al. [18] for the dynamic behaviour of a thick honeycomb sandwich panel subject to thermal loads. Pan et al. noted the central displacement of the heated panel to be strongly dependent on the temperature difference between the front and back surfaces of the structure. In the present study, it was not possible 
to measure this temperature difference because the heating lamps obstructed optical access to one surface of the plate. However, the micro-bolometer was used to measure temperatures on the top surface of the plate, perpendicular to its plane, during one experiment using 4 lamps at full power. The results showed the difference between the temperatures at the front and back edges of the reinforced edge was less than $5 \%$ of the temperatures measured on the front of the plate. This suggests that additional mechanisms, to those postulated by Pan et al., are contributing to the deformation observed in this study. Namely, that the maximum temperature at the centre of the plate, shown in all of the spatial distributions of temperature in Fig. 4, will lead to a larger thermal expansion in the centre than at the edges, which in turn, will cause the plate to buckle out-of-plane to accommodate this local expansion.

In addition, this is exacerbated by the thin plate heating up more quickly than the reinforced-edge, due to the difference in thermal capacities resulting from the difference in thickness; so that, when the plate initially buckles, the reinforced-edge is at a lower temperature and continues to expand due to its rising temperature which relaxes the constraint on the thin plate causing the magnitude of the out-of-plane displacement in the thin plate to be reduced.

Some evidence for this is provided in Fig. 10 where pointwise temperature values at the centre and edges of the plate, when using two lamps, have been plotted as a function of time. The centre of plate was always hottest and transverse edge was always the coolest; however, the temperature differences between the plate's centre and the longitudinal and transverse edges are not constant and exhibit maxima that occur shortly before and after, respectively, the maximum out-of-plane displacement. These differences drive the differential thermal expansion and hence the inplane membrane stress; thus, when the temperature differences are greater the buckling would be expected to be greater and to decrease as the temperature differences reduce to the steady state. This mechanism will operate regardless of whether the initial shape of the plate is convex or concave towards the heat source, as shown by the results in Fig. 11 for the plate in both positions.

Hence, it can be concluded that the in-plane temperature distribution had a significant impact on the magnitude and shape of the deformation, with localised heating causing larger deflections to occur. The finding that the behaviour is more influenced by the distribution of the energy flux rather than the mean magnitude of the flux is likely to be important in designing the skin of structures subject to high heat fluxes. However, other parameters including plate thickness, variation of mechanical properties of the material with temperature and residual stresses introduced during manufacturing, are all likely to influence the behaviour. Nevertheless, the results from this study will be relevant in cases, such as hypersonic flight, where the thermal loading is cyclic and non-uniform, which

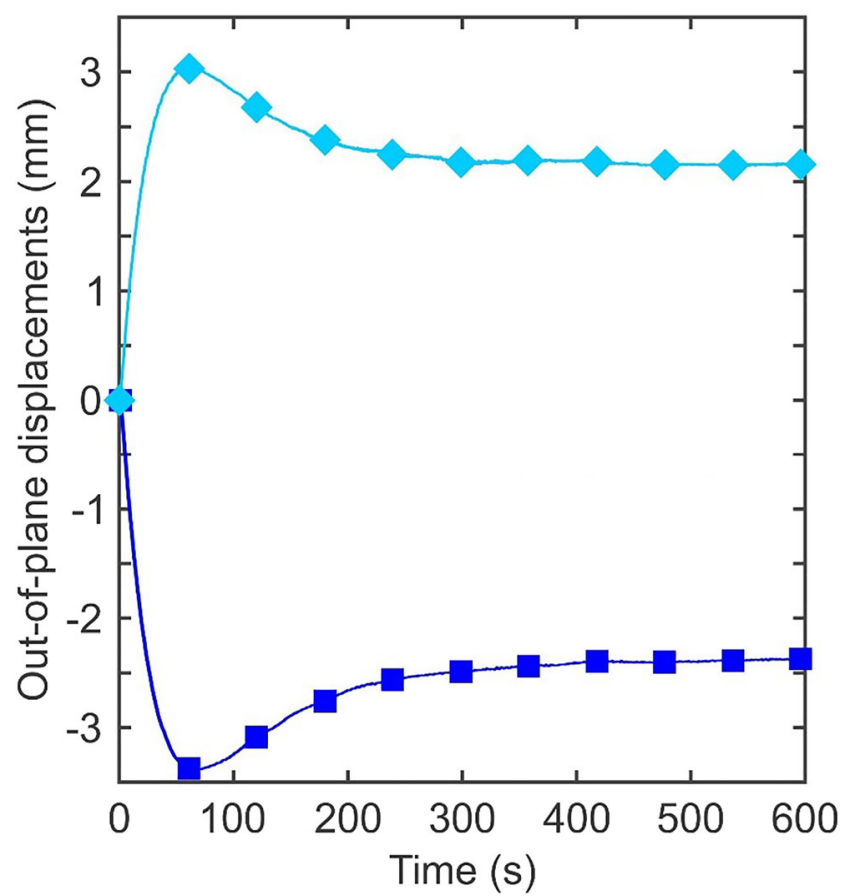

Fig. 11 Point-wise measurements of centre displacement, with rigid body motion removed, for the plate in its original orientation (square markers) with the initial shape convex towards the lamps, as in Fig. 3, and rotated by $\pi$ about its transverse axis (diamond markers) with the initial shape concave toward the lamps

contributes to component fatigue and, eventually, ultimate failure of the structure.

\section{Conclusions}

Thermal loading of an edge-reinforced thin plate has been conducted using multiple configurations of heating lamps and power outputs that achieved maximum temperatures of around $700{ }^{\circ} \mathrm{C}$. Full-field displacement and temperature data were concurrently acquired while thermally loading the plate for $10 \mathrm{~min}$ and during this time period the temperature rose from room temperature to an approximately steady state temperature in about $200 \mathrm{~s}$. The results showed that the reinforced plate behaved as a dynamic system in response to this thermal loading with a post-buckled shape that was dome-shaped.

It was observed that the plate deformation was not proportional to the amount of energy supplied to the plate but dependent on the in-plane temperature distribution because heating of narrow sections across the plate appear to induce substantial compressive membrane stresses that caused out-of-plane buckling. This correlates well with predictions from Mansfield [23] and Liu et al. [4].

Full-field data suggested this behaviour to be at least partially associated with the evolution of temperature difference between the thin plate and its reinforced edges during the loading. The decrease in this temperature gradient reduced 
the differential thermal expansion and hence relaxed the constraint imposed by the reinforced edges on the thin plate, which sprung back until it reached a stable state.

Acknowledgements This effort was sponsored by the European Office of Aerospace Research and Development and the Air Force Office of Scientific Research, USAF under grant numbers FA9550-16-1-0091 and FA9550-16-1-0055. The U.S. Government is authorized to reproduce and distribute reprints of Governmental purpose notwithstanding any copyright notation thereon. Lt. Col. David Garner (EOARD) and Dr. Jaimie S Tiley (AFOSR) are the program officers for these grants respectively. The authors are grateful for further support from the UK's Engineering and Physical Sciences Research Council (EPSRC).

Open Access This article is distributed under the terms of the Creative Commons Attribution 4.0 International License (http:// creativecommons.org/licenses/by/4.0/), which permits unrestricted use, distribution, and reproduction in any medium, provided you give appropriate credit to the original author(s) and the source, provide a link to the Creative Commons license, and indicate if changes were made.

\section{References}

1. Frostig Y, Thomsen OT (2008) Thermal buckling and postbuckling of sandwich panels with a transversely flexible core. AIAA J 46(8): 1976-1989

2. Mead DJ (2003) Vibration and buckling of flat free-free plates under non-uniform in-plane thermal stresses. J Sound Vib 260: 141-165

3. Chen H, Virgin LN (2004) Dynamic analysis of modal shifting and mode jumping in thermally buckled plates. J Sound Vib 278(1-2): 233-256

4. Liu L, Lv B-Y, Li Y-S (2016) Dynamic response of acoustically excited plates resting on elastic foundations in thermal environments environments. Compos Struct 156:35-46

5. Adineh M, Kadkhodayan M (2017) Three-dimensional thermoelastic analysis and dynamic response of a multi-directional functionally graded skew plate on elastic foundation. Compos Part B 125:227-240

6. Nguyen KD, Nguyen-xuan H (2015) An isogeometric finite element approach for three-dimensional static and dynamic analysis of functionally graded material plate structures. Composite Structures 132:423-439

7. Praveen GN, Reddy JN (1998) Nonlinear transient thermoelastic analysis of functionally graded ceramic-metal plates. Int J Solids Struct 35(33):4457-4476
8. Reddy JN (2000) Analysis of functionally graded plates. Int J Numer Meth Engng 47:663-684

9. Vel SS, Batra RC (2003) Three-dimensional analysis of transient thermal stresses in functionally graded plates. Int J Solids Struct 40(25):7181-7196

10. Steeves CA, Mercer C, Antinucci E, He MY, Evans AG (2009) Experimental investigation of the thermal properties of tailored expansion lattices. Int J Mech Mater Des 5:195-202

11. Yamamoto N, Gdoutos E, Toda R, White V, Manohara H, Daraio C (2014) Thin films with ultra-low thermal expansion. Adv Mater 26: 3076-3080

12. Bailey CD (1981) Vibration and local instability of thermally stressed plates. Comput Methods Appl Mech Eng 25(3):263-278

13. Kaldas MM, Dickinson SM (1981) The flexural vibration of welded rectangular plates. J Sound Vib 75(2):163-178

14. Murphy K, Virgin LN, Rizzi SA (1996) Characterizing the dynamic response of a thermally loaded, acoustically excited plate. J Sound Vib 196(5):635-658

15. Jin T, Ha NS, Le VT, Goo NS, Jeon HC (2015) Thermal buckling measurement of a laminated composite plate under a uniform temperature distribution using the digital image correlation method. Compos Struct 123:420-429

16. Yuan W, Song H, Wang X, Huang C (2015) Experimental investigation on thermal buckling behavior of truss-core sandwich panels. AIAA J 53(4):948-957

17. Thornton EA, Coyle MF, Mcleod RN, Thornton EA, Coyle MF, Mcleod RN (1994) Experimental study of plate buckling induced by spatial temperature gradients. J Therm Stresses 17(2):191-212

18. Pan B, Yu L, Wu D (2015) Thermo-mechanical response of superalloy honeycomb sandwich panels subjected to non-steady thermal loading. Mater Des 88:528-536

19. Haynes International (1997) HASTELLOY $® X$ ALLOY. [Online]. Available: http://www.haynes.ch/doc/HASTELLOY_X.pdf. Accessed 25 Jan 2017

20. Chandler HE (1996) Heat treater's guide: practices and procedures for nonferrous alloys. ASM International, Russell Township

21. Pan B, Wu D, Gao J (2014) High-temperature strain measurement using active imaging digital image correlation. J Strain Analysis 49(4):224-232

22. López-Alba E, Sebastian CM, Santos Silva AC, Patterson EA (2018) Experimental study of mode shifting in an asymetrically heated rectangular plate. J Sound Vib 439:241-250

23. Mansfield EH (1962) The effect of temperature variations in the plane and through the thickness of a circular lenticular plate the effect of temperature variations in the plane and through the thickness of a circular lenticular plate. Ministry of Aviation Aeronautical Research Council, HM Stationery Office, Richmond

Publisher's Note Springer Nature remains neutral with regard to jurisdictional claims in published maps and institutional affiliations. 